\title{
Alcohol and COVID-19: How Do We Respond to This Growing Public Health Crisis?
}

\author{
Dawn E. Sugarman, $P h D^{1,2}$ and Shelly F. Greenfield, MD, MPH ${ }^{1,2}$ (]) \\ 'Division of Alcohol, Drugs, and Addiction, McLean Hospital, Belmont, MA, USA; ${ }^{2}$ Department of Psychiatry, Harvard Medical School, Boston, MA, \\ USA.
}

J Gen Intern Med 36(1):214-5

DOI: $10.1007 / \mathrm{s} 11606-020-06321-\mathrm{Z}$

(C) Society of General Internal Medicine 2020

B efore the onset of COVID-19, the USA was experiencing rising rates of alcohol use, high-risk drinking, and alcohol use disorder (AUD) with negative consequences including increases in alcohol-related morbidity and mortality. ${ }^{1}$ As a result of the COVID-19 pandemic, it is likely that the USA will see further increases in alcohol consumption similar to those observed in the $\mathrm{UK}^{2}$ and Australia, ${ }^{3}$ raising significant public health concerns. In the USA, while effective screening, medication, and behavioral treatments are available, only $7.9 \%$ of adults with AUD received any alcohol treatment in 2018. ${ }^{4}$ This compounds the challenge of expanding treatment capacity for those who relapse to harmful drinking or have new onset of alcohol-related problems as a consequence of this pandemic.

Excessive alcohol use is a common response to coping with stress $^{3}$ and increased alcohol use following past traumatic events has been demonstrated in the USA with associated adverse health and social consequences. ${ }^{5}$ A meta-analysis of 31 population-based studies of mass terrorist attacks concluded that $7.3 \%$ of a population can be expected to report increased alcohol consumption in the first two years after the attack, with $20 \%$ probability that the prevalence will be as high as $14 \% .^{5}$

That said, COVID-19 is distinct from other catastrophic events because of the extensive population exposure to ongoing trauma. The mortality rate from COVID-19 has already resulted in large numbers of Americans experiencing loss and grief. There is also widespread social disruption and isolation, while at the same time, social support and access to medical care have been delayed or limited due to stay-at-home orders and COVID-19 restrictions. This pandemic comes with severe domestic and global negative economic impacts and the length and scope of the pandemic are greater than we have seen with natural disasters and terrorist attacks, leading to prolonged

Received June 1, 2020

Accepted October 15, 2020

Published online October 26, 2020 stress and uncertainty. In addition to the psychological stress, stay-at-home orders that are recurring in some states based on increased case rates, uncertainties related to school re-openings, self-isolation, and quarantining can lead to additional stress, which can in turn lead to increased drinking.

According to one study, social isolation during the pandemic lockdown in London was a risk factor for increased alcohol consumption among people with AUD as well as relapse among those previously abstinent. ${ }^{2}$ In an Australian study $(N=4462), 30.8 \%$ of participants endorsed drinking "a lot more than normal" during the pandemic. ${ }^{3}$ This study also identified certain pre-pandemic characteristics that were predictors of increased consumption during the pandemic including pre-pandemic heavy drinking; being a woman, aged 2564 , or in a higher income bracket; and having a history of mental illness. In addition, those who lost jobs as a result of the pandemic were at greater risk for increased drinking as were those who had sleep disruptions, changes in eating behaviors, and higher levels of depression and stress. Similarly, in the USA, there is evidence that psychological distress related to COVID-19 is significantly associated with alcohol use and this relationship is more pronounced for women than men. ${ }^{6}$

Alcohol use and heavy drinking are associated with numerous adverse health consequences including high blood pressure, stroke, liver disease, cancer, and alcohol-impaired accidents and driving fatalities. ${ }^{1,7}$ In addition to the physical effects, heavy alcohol use exacerbates depression and is linked to suicide, contributes to sleep problems, and increases the likelihood of intimate partner violence, child abuse, and neglect. ${ }^{7}$ Alcohol-related health and social consequences are a substantial economic burden in the USA and globally, exacerbating the critical need for intervention in the context of COVID-19.

Are there approaches that can be implemented in the USA to moderate and reduce rising alcohol consumption in the face of this pandemic? Recognition of the population risk of increased alcohol consumption is a first step and the intervention approach must be multifaceted. Public health messaging, early intervention, and access to treatment are key components and include primary care treatment integration, collaborative care coordination, adequate insurance and reimbursement, and expanded use of telehealth and other virtual platforms.

In order to increase awareness and dispel dangerous myths about alcohol and COVID-19, the World Health Organization 
released a fact sheet. ${ }^{7}$ Public health messages in the USA have lagged behind cultural messages promoting alcohol as a way to cope with pandemic-related stress. It is important that public health messages be targeted to include managing stress and anxiety without using alcohol, drinking within safe limits during physical distancing and social isolation, and education about knowing when an individual ought to be concerned about themselves or someone else. ${ }^{7}$

As we consider better public health messaging including the most effective outlets for these messages, we need simultaneously to prepare for a surge in treatment needs across the continuum of severity including drinking-related exacerbation of other co-occurring medical conditions. Overcoming longstanding barriers in primary care to management of patients with alcohol misuse and AUD is especially salient at this time of social adversity. One approach is to increase identification of harmful drinking as part of disease management for comorbid conditions commonly treated in primary care including hypertension, insomnia, depression, and anxiety. ${ }^{8}$ We can add other social adversity risk factors specific to this pandemic that have been associated with increased drinking such as recent job loss due to COVID-19. ${ }^{3}$ In this regard, early intervention based on implementation of integrated alcohol screening in health care as part of treatment for commonly co-occurring conditions will be critical, ${ }^{8}$ including mental health problems frequently encountered in primary care such as anxiety, trauma, and depression. Targeted interventions for those at risk for relapse or exacerbation of use among identified heavy drinkers or those with AUD can also be effective during this pandemic. $^{2}$ In addition, collaborative care models can improve implementation of evidence-based treatment for AUD ${ }^{9}$ especially when supported by adequate reimbursement for care coordination, management, and cross-system integration.

To mitigate the spread of COVID-19, many treatment facilities limited their enrollment capacities, making it harder for some patients to access treatment. ${ }^{10}$ Temporary changes to federal regulations have increased access to telehealth services with potential to increase capacity for treatment. Evidencebased mobile and online programs for managing harmful drinking and AUD could expand the reach of services, especially if made available without cost to patients. Even with the expansion of virtual treatment and online support services, there will be many individuals unable to use these services or in need of a higher level of care. Inpatient and other detoxification programs will need to balance capacity for providing in-person services with safety practices to reduce spread of COVID-19 infection. Ensuring adequate insurance for treatment will be essential with the added concern that many individuals will lose their employer-based health insurance and access to addiction treatment insured by state Medicaid programs varies widely.
The full impact of COVID-19 on alcohol use is not yet known. However, the current rise in drinking during the acute phase of the pandemic mandates a deliberate and clear public health and medical response and a multifaceted approach. The sooner we can intervene to assist individuals in limiting their use of alcohol as a means of coping with this pandemic's myriad stressors, the better chance we have of preventing severe psychosocial and health consequences of overuse of alcohol and an increase in AUD as an important comorbidity of COVID-19.

Corresponding Author: Shelly F. Greenfield, MD, MPH; Division of Alcohol, Drugs, and Addiction, McLean Hospital, Belmont, MA, USA (e-mail: sgreenfield@mclean.harvard.edu).

Funding The authors received support from NIH/NIDA (K23DA050780 (DES).

\section{REFERENCES}

1. White AM, Castle IP, Hingson RW, Powell PA. Using Death Certificates to Explore Changes in Alcohol-Related Mortality in the United States, 1999 to 2017. Alcohol Clin Exp Res. 2020;44(1):178-187.

2. Kim JU, Majid A, Judge R, et al. Effect of COVID-19 lockdown on alcohol consumption in patients with pre-existing alcohol use disorder. Lancet Gastroenterol Hepatol. 2020;5(10):886-887. https://doi.org/10. 1016/S2468-1253(20)30251-X.

3. Neill E, Meyer D, Toh WL, et al. Alcohol use in Australia during the early days of the COVID-19 pandemic: Initial results from the COLLATE project. Psychiatry Clin Neurosci. 2020; https://doi.org/10.1111/pcn. 13099.

4. National Institute on Alcohol Abuse and Alcoholism. Alcohol Facts and Statistics. 2020; https://www.niaaa.nih.gov/sites/default/files/ AlcoholFactsAndStats.pdf. Accessed July 24, 2020.

5. DiMaggio C, Galea S, Li G. Substance use and misuse in the aftermath of terrorism. A Bayesian meta-analysis. Addiction. 2009;104(6):894-904.

6. Rodriguez LM, Litt DM, Stewart SH. Drinking to cope with the pandemic: The unique associations of COVID-19-related perceived threat and psychological distress to drinking behaviors in American men and women. Addict Behav. 2020;110:106532.

7. World Health Organization. Alcohol and COVID-19: What you need to know. Geneva: WHO European Office for the Prevention and Control of Noncommunicable Diseases; 2020.

8. Rehm J, Anderson P, Manthey $\mathbf{J}$, et al. Alcohol Use Disorders in Primary Health Care: What Do We Know and Where Do We Go? Alcohol Alcohol. 2016;51(4):422-427.

9. Watkins KE, Ober AJ, Lamp K, et al. Collaborative Care for Opioid and Alcohol Use Disorders in Primary Care: The SUMMIT Randomized Clinical Trial. JAMA Intern Med. 2017;177(10):1480-1488.

10. Collins F. NIH Directors Blog: Coping with the Collision of Public Health Crises: COVID-19 and Substance Use Disorders. 2020. https:// directorsblog.nih.gov/2020/04/21/coping-with-the-collision-of-publichealth-crises-covid-19-and-substance-use-disorders/. Accessed September 9,2020

Publisher's Note: Springer Nature remains neutral with regard to jurisdictional claims in published maps and institutional affiliations. 